\section{Jean Rouxel (1935-98)}

\section{Pioneer in chimie douce}

Jean Rouxel, who died suddenly on 19 March aged 63, had only a few months previously received the highest award that France can bestow on a scientist - the annual gold medal of the CNRS, the Centre National de la Recherche Scientifique. Rouxel was primarily a solidstate chemist, but had a wide-ranging influence on all aspects of inorganic chemistry and materials science. His main standing was as one of the founding fathers of chimie douce ('soft chemistry').

Until the 1960s, solid-state chemistry was a 'hard chemistry' that involved the use of very extreme conditions principally high temperatures - for the synthesis of compounds; such conditions are necessary to overcome the kinetic barriers to diffusion and reactivity, and lead to a unique materials phase that is stable in the chosen conditions. Chimie douce, by contrast, is carried out at low temperature and allows the synthesis of very many atomic arrangements; these are often metastable but have a large variety of structures and properties.

Rouxel initiated a part of chimie douce devoted to intercalation-deintercalation processes with low-dimensional solids. Such solids can be regarded as stacks of infinite one-dimensional or twodimensional molecules, in chains or sheets respectively, and they constitute a fruitful intermediate between molecular inorganic chemistry and solid-state chemistry.

The approach resulted in the discovery of many new compounds, especially among the chalcogenides which are composed of a chalcogen, such as sulphur, selenium or tellurium, and a more metallic element. But it also revealed new concepts, physical effects and chemical properties, and has applications in fields as various as battery design, catalysis and electrochromism (in which pigments change colour when an electric field is applied, the compounds concerned being used in displays).

Rouxel was born in 1935 in a small village in Brittany, and throughout his life remained strongly influenced by Breton customs and culture. He obtained his early scientific education in Brest and later at the University of Rennes, where he met a young, newly appointed professor in inorganic chemistry, Paul Hagenmuller. It was with Hagenmuller that Rouxel discovered and embraced with eagerness the joys - and the attendant doubts - of a research career. Appointed an assistant lecturer in 1957, a few years later he followed Hagenmuller and his group to Bordeaux, obtaining his $\mathrm{PhD}$ there in 1961. At that time, Nantes became an independent university and appointed Rouxel a professor in 1963. There he created his own laboratory, gathering a cadre of young and energetic colleagues around him. Fifteen years later, the group received the support of the CNRS, attaining the status of associate laboratory; finally, in 1988, the Institute of Materials at Nantes was created with Rouxel as head. This institute was and remains a model of interdisciplinarity, where solid-state and molecular chemists, solid-state physicists and other specialists in materials science all rub shoulders.

Rouxel's scientific achievements stemmed from the originality of his work on chemical bonding in low-dimensional solids, materials which he manipulated as if they were sheets of paper in a book between which he could insert various molecules. Most usually, the molecules concerned are cations, which donate electrons to the sheet and thereby reduce it. This reduction of the sheet by increasing the number of $d$ electrons per metal atom sometimes leads to metal-metal bonding (like that observed in clusters), and sometimes to electronic delocalization in bands (like that seen in metals). Occasionally an intermediate situation occurs, as it does in niobium selenide, with the formation of charge density waves. Rouxel viewed these in a fresh light, as bonding density waves, thereby showing that physicists and chemists are often looking at the same phenomena with complementary eyes.

The reverse process, deintercalation of cations out of the sheets, of course involves oxidation and is one of the most powerful synthesis processes in chimie douce. But it raises some difficult questions. For instance, from which band do the electrons that are lost from the sheet come - the metallic $d$ orbitals or the $s p$ anionic bands? Rouxel showed that what matters is the relative energies of the electron bands. For example, with some pairings of metal and chalcogen, such as iridium with tellurium, it is
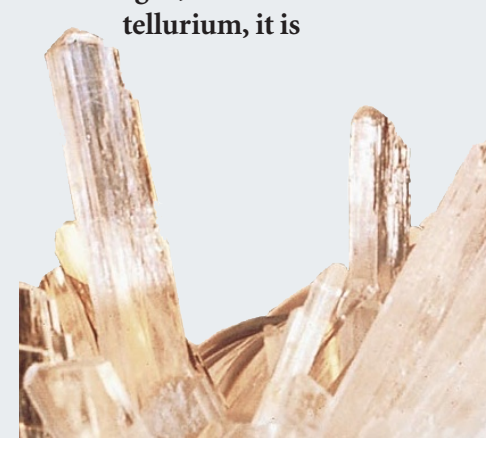

possible to empty the $p$ anionic band first; this creates holes in place of the antibonding electrons, which stabilizes the tellurium-tellurium anionic bonds. This is equivalent to adding bonding electrons to an empty band - both promote bonding. This chemistry of antibonding electrons was the final area to be developed by Rouxel during his highly fruitful career.

Before that, he had tackled another fundamental question to do with so-called 'misfit' structures. These are twodimensional solids with two kinds of sheet but only one common crystallographic parameter, a peculiar property which marks a conceptual limit to the basic notion of a thermodynamically welldefined phase.

Jean had an endearing personality, being both modest and down-to-earth, yet capable of great enthusiasm - he took the same intense interest in the roses in his garden as in being an ambassador for French science at international meetings. He also always liked to have young scientists around him, and closely followed their scientific upbringing. During the 1970 s, he created a summer school where the oldest students taught the youngest ones the basic concepts and methods of solid-state chemistry, informally and in the friendliest of atmospheres. The first meeting took place in Brittany, not too far from Nantes, and was named after a nearby, moorland village, Galerne. A saying of the local farmers, "The wind is from Galerne", means that an invigorating wind is blowing from the west, from the direction of the steeple of Galerne's church. One might say the same of Jean Rouxel his was an invigorating influence on solidstate chemistry that blew steadily from the west of France.

\section{Michel Pouchard}

Michel Pouchard is in the Institut de Chimie de la Matière Condensée de Bordeaux - CNRS, Avenue du Dr A. Schweitzer, 33608 Pessac Cedex, France. e-mail:pouchard@icmcb.u-bordeaux.fr

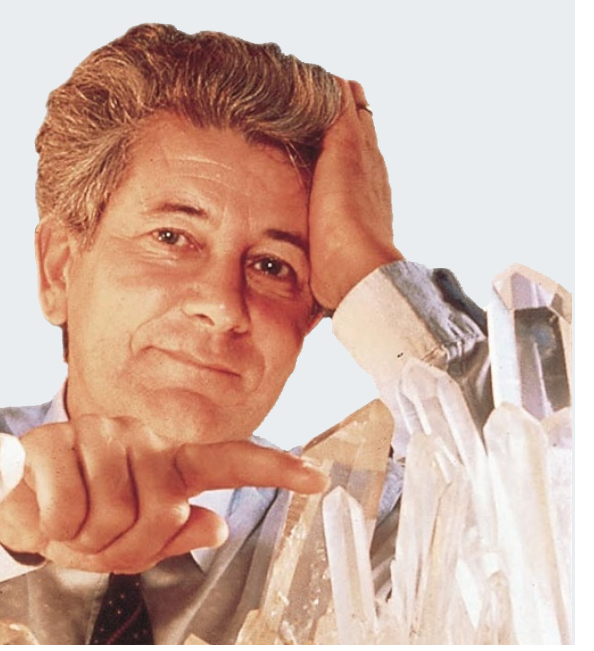

